\title{
Radial basis function neural network in fault detection of automotive engines
}

\author{
Adnan Hamad ${ }^{1}$, Dingli Yu ${ }^{1}$, J. B. Gomm ${ }^{1}$, Mahavir S. Sangha ${ }^{2}$ \\ ${ }^{I}$ Control System Research Group, School of Engineering, Liverpool John Moores University, Byrom Street, Liverpool L3 3AF, U.K \\ ${ }^{2}$ Test Technology \& Emissions, Cummins Inc., Daventry NN11 8NU, U.K \\ Correspondence author abohliga@yahoo.com
}

\begin{abstract}
Fault detection and isolation have become one of the most important aspects of automobile design. A fault detection (FD) scheme is developed for automotive engines in this paper. The method uses an independent Radial Basis Function (RBF) Neural Network model to model engine dynamics, and the modelling errors are used to form the basis for residual generation. A dependent RBFNN model is a model which uses output data of a plant as a target output then use it to train the neural network, while, The independent RBFNN model is a higher accuracy than the dependent model and the errors can be detected by this model, this is because this model does not dependent on the output of the plant and it will use its output as a target, so if any faults in the plant will be not effect in the model and this faults will be detected easily and clearly. The method is developed and the performance assessed using the engine benchmark, the Mean Value Engine Model (MVEM) with Matlab/Simulink. Five faults have been simulated on the MVEM, including three sensor faults, one component fault and one actuator fault. The three sensor faults considered are 10-20\% change superimposed on the outputs of manifold pressure, temperature and crankshaft speed sensors; one component fault considered is air leakage in intake manifold and Exhaust Gas Recycle (EGR); the actuator fault considered is the malfunction of fuel injector. The simulation results showed that all the simulated faults can be clearly detected in the dynamic condition throughout the operating range.
\end{abstract}

Keywords: Automotive engine, independent RBFNN model, RBF neural network, fault detection.

\section{Introduction}

A fault is any type of malfunction of components that may happen in a system and this fault will degrade the system performance. Fault detection is the program which informs us that something wrong in the system and needs to be repaired. Also, fault isolation is way to determine which fault occurs among the possible faults. Over the last few years, many different fault detection and isolation methods have been proposed. To detect faults we usually compare the outputs of the real system which is in this paper the mean value engine model, and the outputs of a neural network model of the engine. Because a neural network is capable of approximating a nonlinear function to any desired degree of accuracy, it is used as a model of a dynamic system. The modelling error is then used as the residual for fault detection (Kimmich et al., 2005). Furthermore, a neural network can also be trained to isolate different faults (Capriglione et al., 2004). Sorsa et al. (1991) investigated a number of possible neural network architectures for fault diagnoses. The multilayer perceptron network with a hyperbolic tangent as the nonlinear element was reported to be best suited for the task. Yu et al. (1999) proposed RBF neural networks for process fault diagnosis. The use of the output prediction error, between a neural network model and a non-linear dynamic process, as a residual for diagnosing actuator, component and sensor faults was analysed. Jamsa-Jounela et al. (2002) had described a fault diagnosis system and discussed some application results from the Outokumpu Harjavalta smelter. Demetgul et al. (2009) investigated fault diagnosis of pneumatic systems with artificial neural network algorithms. A pneumatic manufacturing system was simulated with modular production system (MPS) and automated monitoring of the system was considered. FDI for automotive engines has been investigated for more than two decades. Isermann (2005) has proposed model-based fault-detection and diagnosis methods for some technical processes. The goal was to generate several symptoms indicating the difference between nominal and faulty status. Based on different symptoms fault diagnosis procedures follow, determining the fault by applying classification or inference methods. His contribution gave a short introduction into the field and showed some applications for an actuator, a passenger car and a combustion engine. For fault 
diagnosis of Diesel engines three detection modules are proposed to generate symptoms based on mainly production- type sensors. The symptoms are generated with nonlinear output error and input error parity equations for special model-based characteristic quantities like volumetric efficiency, oscillations of pressure, flow and (not shown here) for angular speed and oxygen content. In many critical applications like nuclear plants, aircrafts, space vehicles and chemical processes, the use of fault tolerant measurement systems is strongly required. Thus, the hardware and/or software instrument fault detection, isolation and accommodation (IFDIA) schemes are more and more widespread in many contexts. Automotive is one of this, since in the last decade, private and public transportation vehicles have begin equipped with a lot of a sensor-based electronic systems devoted to grant the passenger safety and comfort (Anti-lock braking system, Anti-spin regulation, Electronic stability program, Airbag, air conditioning, and so on) as well as to control fuel injection and ignition and the pollution emissions of the engines. On-line Sensor fault Detection, isolation, and accommodation in automotive engines had studied by Capriglione et al. (2004). Their paper was described the hybrid solution, based on artificial neural networks (ANNs), and the production rule adopted in the realization of an instrument fault detection, isolation, and accommodation scheme for automotive applications. The fault accommodation has shown a good performance with maximum error of 5\%. Fault detection for modern Diesel engines using signal- and process model-based methods have been proposed by Kimmich et al. (2005). Their contribution showed a systematic development of fault detection and diagnosis methods for two system components of Diesel engines, the intake system and the injection system together with the combustion process. The residuals were generated by applied semiphysical dynamic process models, identification with special neural networks, signal models and parity equations. The deflection of the residuals allowed the detection and diagnosis of different faults. Further residuals were developed for the exhaust system. The additional symptoms increase the fault detection coverage. Wu et al. (2009) in their paper had proposed an expert system for fault diagnosis system in internal combustion engines using wavelet packet transform (WPT) and ANNs techniques. To verify the effect of the proposed generalized regression neural network (GRNN) in fault diagnosis, a conventional back-propagation network (BPN) was compared with a GRNN network. The experimental results showed the proposed system achieved an average classification accuracy of over 95\% for various engine working conditions.

\section{Spark ignition (SI) engine modelling using RBF neural networks}

The first step in the engine modelling by using RBFNN is the generation of a suitable training data set. As the training data will influence the accuracy of the neural network modelling performance, the objective of experiment design on training data is to make the measured data become maximally informative, subject to constraints that may be at hand. A set of random amplitude signals (RAS) were designed for the throttle angle position and the fuel mass flow to obtain a representative set of input data. The ranges of these excitation signals were bounded between 20 and 60 degrees for the throttle angle position and between 0.0005 and $0.003 \mathrm{~kg} / \mathrm{s}$ for the fuel mass flow. Before training or validating the neural network using RAS, all inputs and outputs data obtained from MVEM by simulation will be scaled to the range of $[0,1]$ in order to increase the accuracy of the neural network and decrease the error. The linear scale is used by equations 1 and 2 (Zhai et al., 2007)

$$
\begin{gathered}
u_{S}(k)=\frac{u(k)-u_{\min }}{u_{\max }-u_{\min }} \\
y_{S}(k)=\frac{y(k)-y_{\min }}{y_{\max }-y_{\min }}
\end{gathered}
$$

Where $u_{\min }$ and $y_{\min }$ are the minimum inputs and outputs, also, $u_{\max }$ and $y_{\max }$ are the maximum inputs and outputs among the data set, while $u_{\mathrm{s}}$ and $y_{\mathrm{s}}$ are the scaled input and output respectively. The first 300 samples of excitation signals for the throttle angle position and fuel injection, which are engine model inputs are shown in Figures 1 and 2. At the beginning the RBF neural network will be trained and tested, so, it will receives five inputs signals, which are manifold pressure, temperature, crankshaft speed, the throttle angle and the fuel mass flow and has three outputs, manifold pressure, temperature, crankshaft speed. By using K-means algorithm and $\rho$ - Nearest Neighbours method the width in hidden layer nodes of the RBF neural network $\sigma$ and the centres $c$ are calculated. For train the weights $w$ of the RBF neural networks, the recursive least square algorithm was applied and the following data are used: $\mu=0.98, w(0)=1.0 \times 10^{-6} \times U_{(n h \times 3),} P(0)=1.0 \times 10^{8} \times I_{(\mathrm{nh})}$. Where $\mu$ is called the forgetting factor ranging from 0 to $1, I$ is an identity matrix and $U$ is an ones matrix. To make this neural network behave as an independent model, the neural network model will use only the first three rows of the MVEM output matrix which are contain values of manifold pressure, manifold temperature and crankshaft speed, after that the output of the neural network will be used as a target matrix to train and test this neural network. The excitation signal to generate training data covers the whole possible operating points and then the training data was collected therefore the train model will be valid for all the operating point. The engine data was divided into two parts, the first 4000 data samples is used for training neural network and the 2000 data samples is used for neural network model validation, This procedure in order to confirm validity of the neural network model. The Figure 3, a,b and c shows the simulation result of the engine model output (speed, pressure and temperature respectively) and the RBF neural networks output during 100 samples for training and 100 samples for the test. It can be seen that the perfect equivalent between the two outputs with a very stingy error, in 
general, the modelling error of the training data set is often smaller than the test data set. The mean absolute error (MAE), which is given by equation (3) was used evaluate the modelling.

$$
M A E=\frac{1}{N} \sum_{K=1}^{N}|f(k)-y(k)|=\frac{1}{N} \sum_{K=1}^{N}|e(k)|
$$

The MAE is an average of the absolute errors $e(k)=f(k)-y(k)$ (Zhai et al., 2007). For modelling performance, $f(k)$ is the prediction by neural network model and $y(k)$ the output of SI engine. For this model the MAE values of crankshaft speed, manifold pressure and temperature are $0.0069,0.0155$ and 0.0059 respectively. Figure $4, \mathrm{a}, \mathrm{b}$ and c shows the 200 samples of the error signals between the engine model and the RBF neural networks for the three outputs, which are crankshaft speed, manifold pressure and temperature respectively.

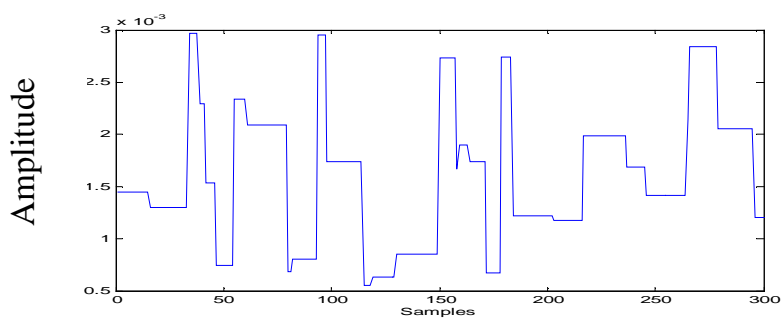

Figure 1. Random Amplitude Signal of the Throttle Angle Position.

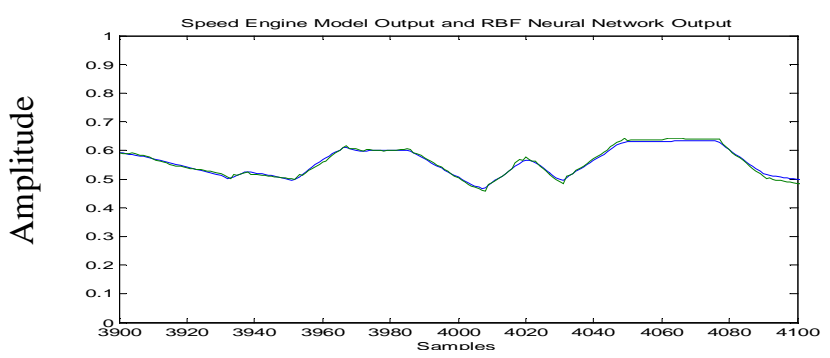

(a)

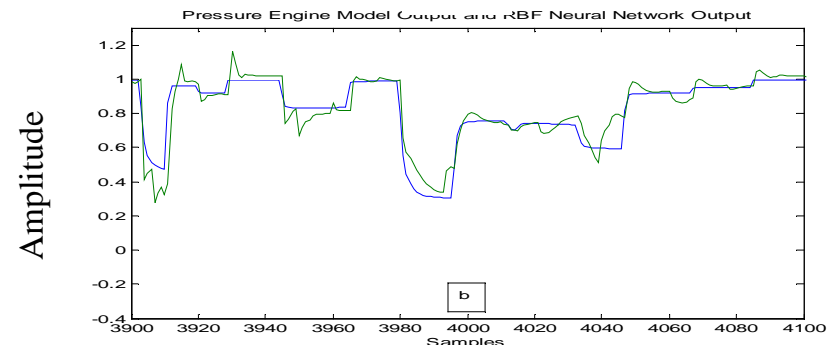

(b)

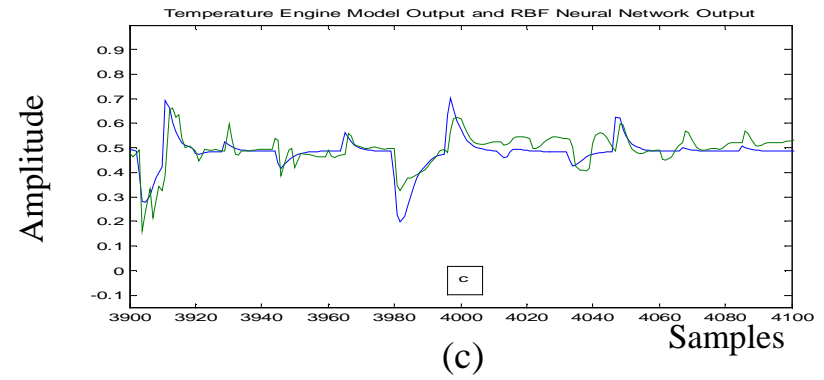

Figure 3, a, b and c. The simulation result of the speed, pressure and temperature engine model output and the RBF neural networks output respectively.

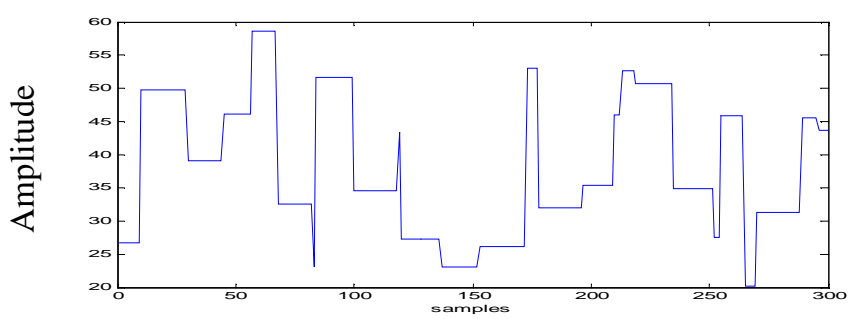

Figure 2. Random Amplitude Signal of the Fuel Injection.

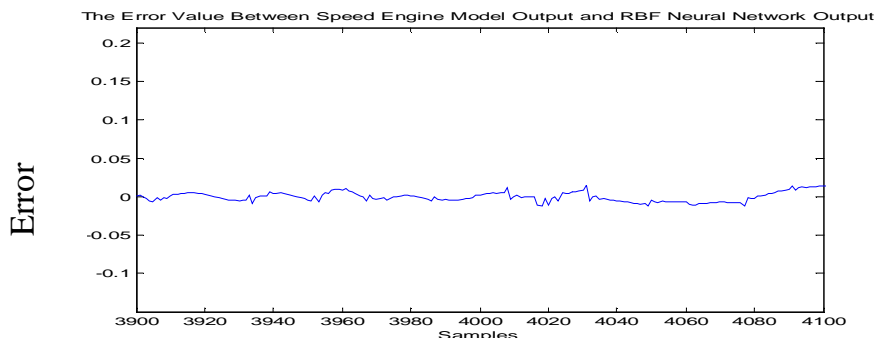

(a)

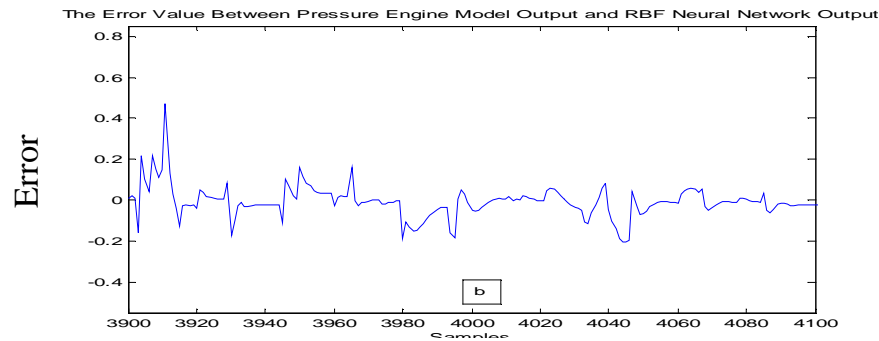

(b)

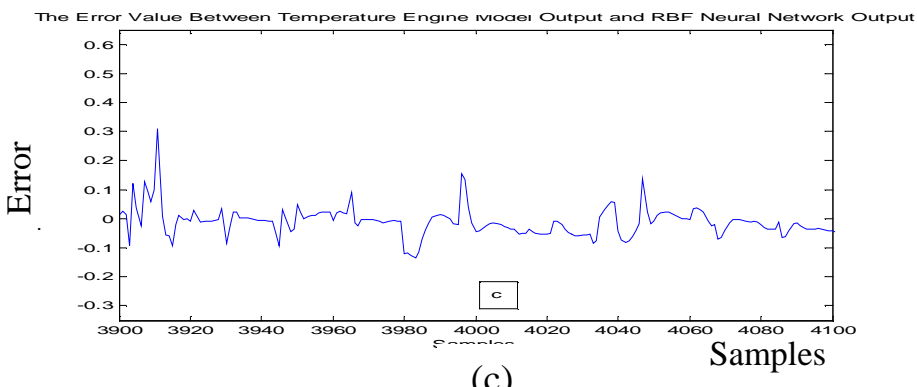

(c)

Figure 4, a, b and c. The error signals between the speed, pressure and temperature engine model output respectively and the RBF neural networks. 
3.1 Air leakage fault: To collect the engine data subjected to the air leakage fault, the equation (4) (Hendricks et al., 2000) of the manifold pressure is modified to equation (5):

$$
\begin{aligned}
& \dot{p}_{i}=\frac{T_{i} R}{V_{i}}\left(-\dot{m}_{a p}+\dot{m}_{a t}+\dot{m}_{E G R}\right) \\
& \dot{p}_{i}=\frac{T_{i} R}{V_{i}}\left(-\dot{m}_{a p}+\dot{m}_{a t}+\dot{m}_{E G R}-\Delta l\right)
\end{aligned}
$$

Where $\dot{p}_{i}$ is absolute manifold pressure (bar), $\dot{m}_{a t}$ is air mass flow past throttle plate (kg/sec), $\dot{m}_{a p}$ is air mass flow into intake port $(\mathrm{kg} / \mathrm{sec}), \dot{m}_{E G R}$ is EGR mass flow (kg/sec). $\Delta l$ is used to simulate the leakage from the air manifold, which is subtracted to increase the air outflow from the intake manifold. $\Delta l=0$ will represent no air leakage in the intake manifold. The air leakage levels are simulated as $20 \%$ of total air intake in the intake manifold. This fault occurs from sample number 5401 to 5700 , which means from second 108.02 to second 114, see Figure 5.

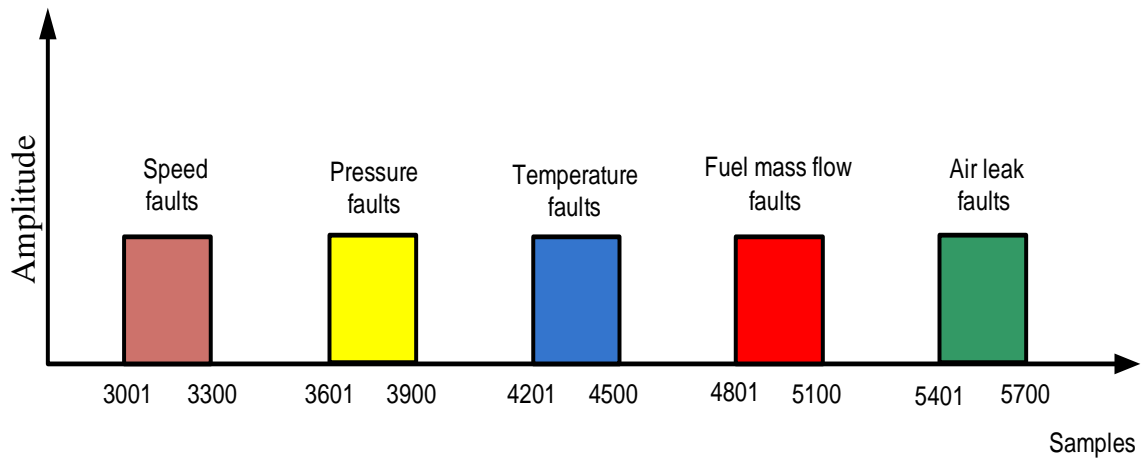

Figure 5. The simulated faults.

3.2 Injected fuel mass fault: For SI engines, in term of control engineering, the target is to achieve an air-fuel mixture with a ratio of $14.7 \mathrm{~kg}$ air to $1 \mathrm{~kg}$ fuel. This means the normal value of air fuel ratio is 14.7 . This value will be changed if there are faults on the fuel injector and the amount of fuel. The value of fuel is regulated as $20 \%$ of the total fuel mass flow from sample number 4801 to 5100, see Figure 5.

3.3 Speed, temperature and pressure sensor faults: Speed, pressure and temperature sensor faults considered are 10-20\% change superimposed on the outputs of crankshaft speed, manifold pressure and temperature sensors. These faults are simulated from sample number 3001 to 3300, from sample number 3601 to 3900 and from sample number 4201 to 4500 respectively. The faulty data for the sensors is generated using multiplying factors (MFs) of 1.1 and 1.2 for the above over -reading respectively, see Figure 5. Faulty data are generated by the Modified MVEM with throttle angle at different values between $20^{\circ}$ and $60^{\circ}$ for all the fault conditions. The 5 states with their MFs are given in Table 1. The engine data for the simulated faults and no fault condition covers almost all transient states of the engine dynamics.

Table 1. The faults states and multiplying factors.

\begin{tabular}{|c|c|c|}
\hline No & Fault Name & MFs \\
\hline 1 & Air Leak 20\% & \\
\hline 2 & Injected fuel mass flow 20\% & \\
\hline 3 & Speed sensor 10\% over reading & 1.1 \\
\hline 4 & Pressure sensor $20 \%$ over reading & 1.2 \\
\hline 5 & Temp. sensor $10 \%$ over reading & 1.1 \\
\hline
\end{tabular}

\section{Fault detection}

The Figure 6 shows the flow chart of fault detection. The RBF neural network receives five inputs signals, the first three inputs signals are manifold pressure, temperature and crankshaft speed which containing fault information, and the second two inputs signals are the throttle angle and the fuel mass flow and has three outputs with each indicating one of the investigated states in 
Table 1. This neural network will use at the beginning only the first three rows of the MVEM output matrix which consists of signals values of manifold pressure, manifold temperature and crankshaft speed, all these three inputs contain sensor, component and actuator faults, after that the output of the neural network will be used as a target matrix, that means this neural network is an independent model. The information flow for the fault detection is illustrated in Figure 7. The hidden nodes which are chosen by k-means method are 12, this is because the test result is very good and the size of neural network will be small, consequently the train and test time will be small. Width and weights are trained using $\rho$-nearest neighbours algorithms and the same data of $\mu$, $w(0), P(0)$ which were used to train neural network engine model are used here. The trained network is then tested for all faults occurring. Figure 8 shows the test results for fault detection for all faults (sensor, component and actuator) before filtering, it can be seen the errors are big. After the filtering operation the results were very good and the errors decreased, see Figure 9 . The detection thresholds are chosen as $0.2, \pm 0.1$ and 0.1 for crankshaft speed, manifold pressure and manifold temperature error signals respectively. High thresholds may lead to missed detections whilst low thresholds will cause false alarm. Thresholds are chosen as $0.2, \pm 0.1$ and 0.1 by utilising experience in minimising false alarm rate.

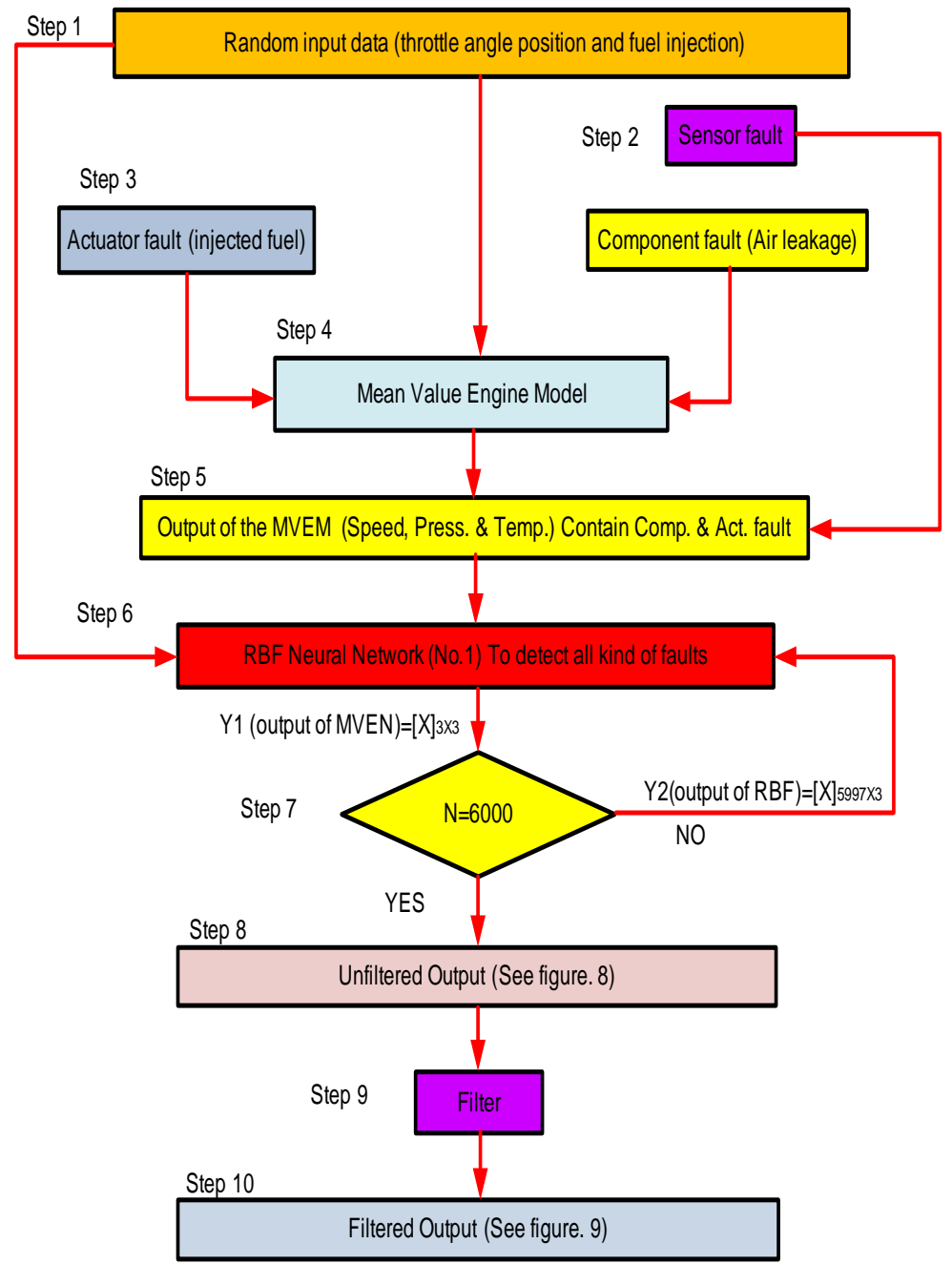

Figure 6. Flow chart of fault detection. 


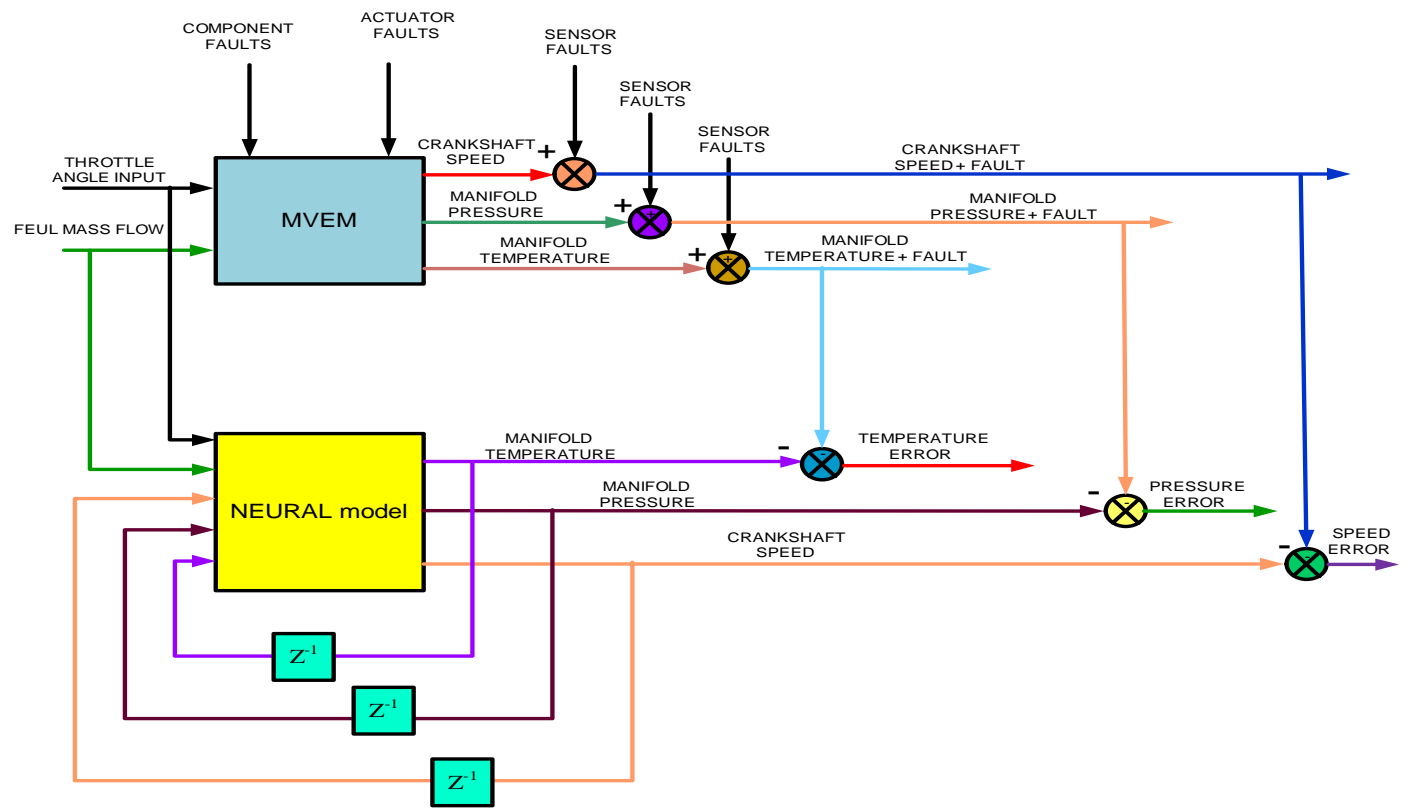

Figure 7.The information flow for the fault detection.

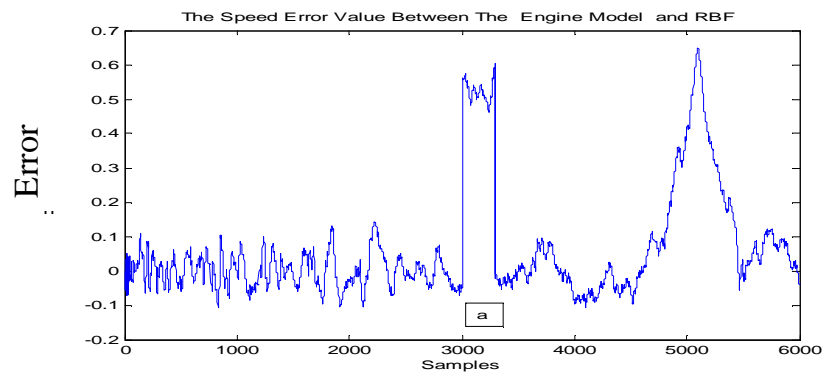

(a)

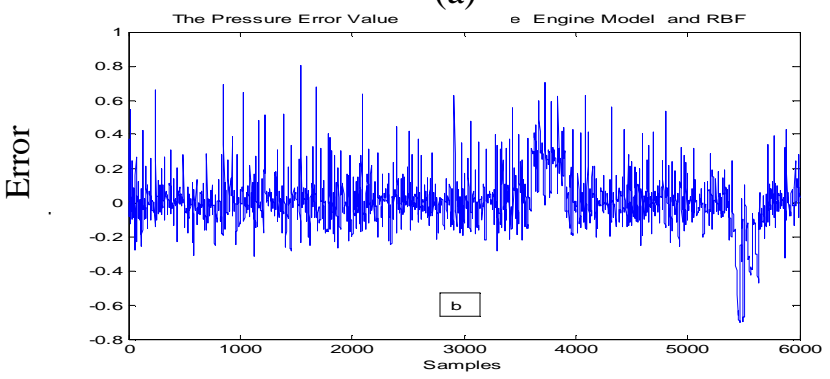

(b)

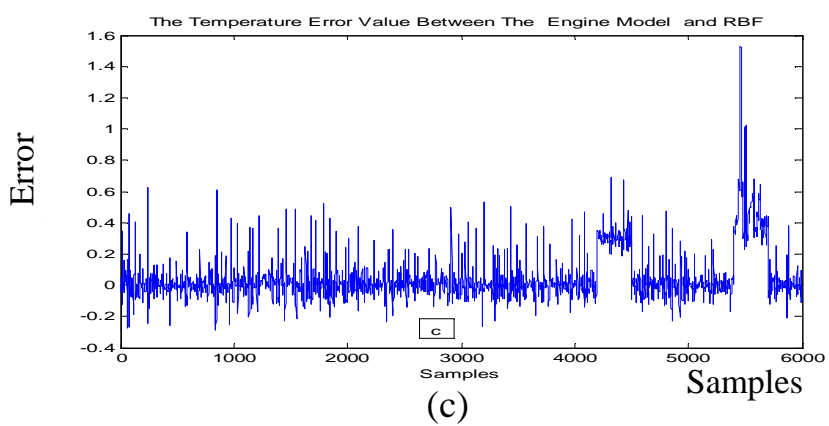

Figure 8. $a, b$ and c. Test results of the fault detection of speed, pres. \& tem. respectively before filtering.

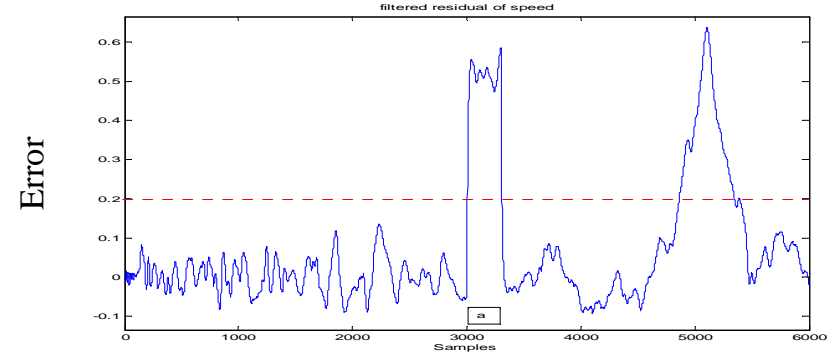

(a)

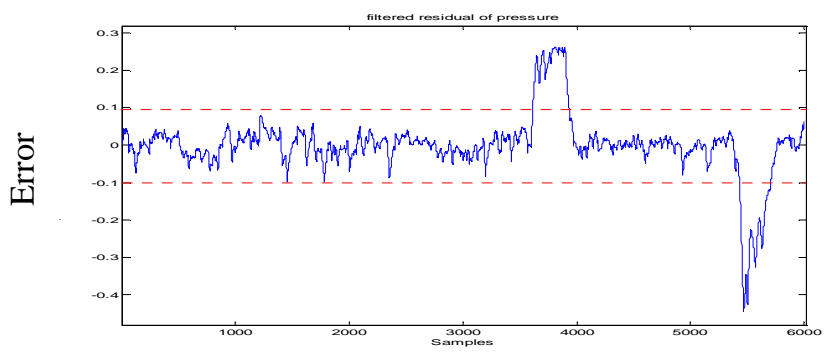

(b)

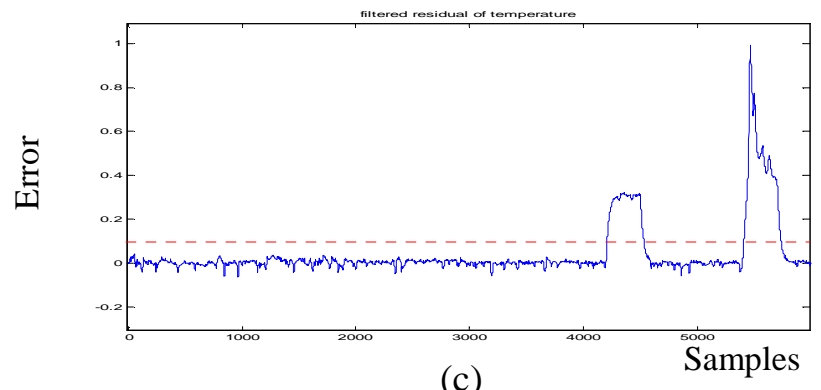

Figure 9. a, b, c. Test results of the fault detection of speed, pres. \& tem. respectively after filtering. 
Figure 10 shows the residual error (re) which is generated by the equation (6)

$$
r e=\sqrt[2]{e n^{2}+e p^{2}+e t^{2}}
$$

Where $e_{n}, e_{p}$ and $e_{t}$ are the error vectors of the speed, pressure and temperature respectively between the engine model and the RBF neural network. Thresholds are chosen as 0.18 , If the residual greater than threshold that means the fault occurs.

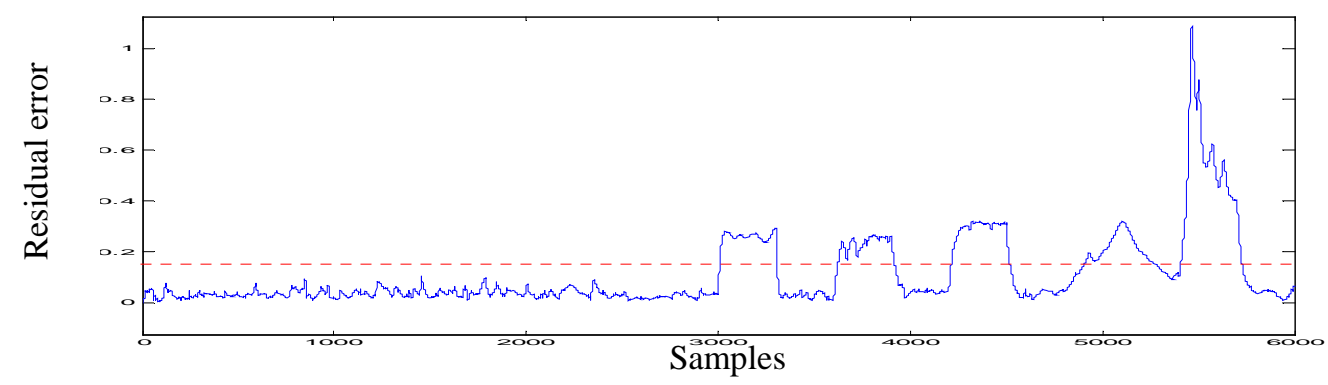

Figure 10. The residual error of the fault detection after filtering with 12 hidden nodes.

\section{Simulation results}

We can summarize the results in the following points:

5.1 Train and Test the Neural Network: The simulation results of train and test by using 12 hidden nodes were very good and the good match between the engine model output and the RBF neural network output was done, (see Figures 3 and 4 ) and the mean absolute errors between them are shown it Table 2 .

Table 2. The Mean Absolute Error (MAE) between the Engine Model Output and the RBF Neural Network Output

\begin{tabular}{|c|c|}
\hline Outputs & MAE \\
\hline Crankshaft speed & 0.0016 \\
\hline Pressure & 0.013 \\
\hline Temperature & 0.0047 \\
\hline
\end{tabular}

5.2 Detect the sensor, components and actuator faults: The test results for fault detection for all kinds of faults were before and after filtering operation were done with 12 hidden nodes, and all the faults were detected after filtering were very clear (see Figure 8) than faults before filtering (see Figure 9) and the detection thresholds were chosen as 0.2 for crankshaft speed, \pm 0.1 for manifold pressure and 0.1 for manifold temperature.

\section{Conclusions}

The MVEM developed by Hendricks and et al (2000) is used for simulations during the research period after small modification. Expansion work has been done to the existing MVEM simulation by including air fuel ratio sensor time delay, temperature sensor dynamics etc. Three sensor faults (intake manifold pressure, temperature and speed), one component fault (leakage in the intake manifold) and one actuator fault (injected fuel mass flow) have been simulated when the simulation model is subjected to disturbances and noise. An independent RBF neural network model was used to model engine dynamics and the training algorithms are reviewed and derived. By using $\rho$ - Nearest Neighbours method and K-means algorithm the width in hidden layer nodes of the RBF neural network $\sigma$ and the centres $\mathrm{c}$ are calculated for RBFNN. The recursive least square algorithm was applied for training the weights $w$ of the RBFNN. Fault detection for engine studied in this paper is using neural network modelling method, this method can detect dynamic faults, and this is because the modelling is for dynamic system, so can detect the faults in 
dynamic condition and for other simulated three types of fault (sensor, actuator and component). From The simulation results it can be seen that the independent RBF neural networks were able to detect sensor, actuator and component faults clearly.

\section{References}

Capriglione, D., Liguori, C., Pianese, C., and Pietrosanto, A. 2004. On-line sensor fault detection, isolation, and accommodation in automotive engines, IEEE Transactions on Instrumentation and Measurement, Vol. 52, No. 4, August, pp.1182-1189.

Demetgul, M., Tansel, I.N. and Taskin, S. 2009. Fault diagnosis of pneumatic systems with artificial neural network algorithms. Expert Systems with Applications Vol. 36. pp. 10512-10519.

Hendricks, E., Engler, D. and Fam, M. 2000. A generic mean value engine model for spark ignition engines. In Proceeding of $41^{\text {st }}$ Simulation Conference, Denmark,. DTU Lyngby.

Isermann, R. 2005. Model-based fault-detection and diagnosis status and applications, Annual Reviews in Control, Vol. 29, pp 7185.

Jamsa-Jounela, S.-L., Vermasvuori, M. Enden, P. and Haavisto, S. 2003. A process monitoring system based on the Kohonen selforganizing maps, Control Engineering Practice, Vol. 11, pp. 83-92.

Kimmich, F. Schwarte, A and Isermann, R. 2005. Fault detection for modern Diesel engines using signal- and process modelbased methods, Control Engineering Practice, Vol. 13, pp.189-203.

Sorsa, T. , Koivo, H.N. and Koivisto, H. 1991. Neural networks in process fault diagnosis. IEEE Transactions on systems, man, and cybernetics, Vol.21, No. 4, July/august

Wu, J. D. and Liu, C. H. 2009. An expert system for fault diagnosis in internal combustion engines using wavelet packet transform and neural network. Expert Systems with Applications, Vol. 36, pp. 4278-4286.

Yu D.L., Gomm, J.B. Williams, D. 1999. Sensor fault diagnosis in a chemical process via RBF neural networks, Control Engineering Practice, Vol. 7, pp. 49-55.

Zhai Y. J. and D. L Yu. 2007. Radial Basis Function Based Feeddback Control for Air Fuel Ratio of Spark Ignition. Proc. Instn Mech. Engrs, Part D: J. Automobile Engineering, Vol. 222, pp. 415-428.

\section{Biographical notes}

Adnan Hamad received his B.Eng. degree in Electrical and Electronic Engineering from bright star university of technology, Libya, in 1994. And his M.Sc. in automatic control from Tabbin institute for metallurgical studies, Egypt, in 2006. He is now a PhD student in school of engineering at Liverpool John Moores University (LJMU), UK. His research interests include fault detection and isolation, process monitoring and neural network

Dingli Yu received the B. Eng. Degree from Harbin University of Civil Engineering, China, in 1982, the master degree from Jilin University of Technology (JUT), China, in 1986, and the Ph. D. degree from Coventry University, UK, in 1995, all in electrical engineering. He was a lecturer at JUT from 1986 to 1990 before he came to University of Salford as a visiting researcher in 1991. He then worked at Liverpool John Moores University as a post-doctoral researcher since 1995 and became a lecturer in 1998, where he is currently a professor of control systems. His research interests include fault detection and fault tolerant control of bilinear and nonlinear systems, adaptive neural networks and their control applications, and model predictive control for chemical processes and engine systems.

J. Barry Gomm received the B. Eng. first class degree in electrical and electronic engineering in 1987 and the Ph. D. degree in process fault detection in 1991 from Liverpool John Moores University (LJMU), UK. He joined the academic staff at LJMU in 1991 and is a reader in intelligent control systems. His research interests include neural networks for modeling, control and fault diagnosis of non-linear processes, intelligent methods for control, system identification, adaptive systems, chemical process, and automotive applications.

M. S. Sangha is a widely experienced Chartered Engineer who is also a certified Six Sigma green belt. He received his BEng first class degree in electrical engineering in 1991 from Jai Narayan Vyas University, Jodhpur, India. He worked as an Electrical Engineer for the largest electricity distribution company (JVVNL) of Rajasthan State in India for about twelve years before perusing his Masters and PhD degrees from the UK. He received an MSc (Intelligent Control) with distinction in 2005 and subsequently a PhD degree in Intelligent Fault Diagnosis in 2008 from Liverpool John Moores University (LJMU), UK. He joined as Principal Engineer in Cummins Engine Co Ltd in 2008 where he is currently working. He is an Editorial Board Member of the International Journal of Engineering, Science and Technology (IJEST). Currently he is managing a special issue for the Journal. He has published several research papers in international journals and conference proceedings. Dr Sangha is a member of the IET and IEEE, and has served as reviewer for several conferences. His current research interests include neural networks for modeling, control and fault diagnosis of non-linear processes, process optimization, adaptive neural networks, intelligent methods for control, system identification, supervisory control and data acquisition (SCADA).

Received July 2010

Accepted November 2010

Final acceptance in revised form December 2010 